\title{
Gene therapy research at the Frontiers of viral immunology
}

\author{
Brad E. Hoffman ${ }^{*}$, Hildegund C. J. Ertl ${ }^{2}$, Cox Terhorst ${ }^{3}$, Katherine A. High ${ }^{4,5}$ and Roland W. Herzog \\ Division of Cellular and Molecular Therapy, Department of Pediatrics, University of Florida College of Medicine, Gainesville, FL, USA \\ 2 Immunology Program, Wistar Institute, Philadelphia, PA, USA \\ ${ }^{3}$ Division of Immunology, Beth Israel Deaconess Medical Center, Boston, MA, USA \\ ${ }^{4}$ Department of Pediatrics, The Children's Hospital of Philadelphia, University of Pennsylvania Medical Center, Philadelphia, PA, USA \\ ${ }^{5}$ Howard Hughes Medical Institute, Philadelphia, PA, USA \\ *Correspondence: bhoffman@ufl.edu
}

Although originally conceived in the mid 1970 s as an alternative to transfection (Goff and Berg, 1976), the use of viral vectors as a tool for clinical gene therapy did not emerge until the 1980s. As Li and Ertl (2011) recapitulate in their thought provoking perspective, viruses meet all the requirements needed for gene therapy. Evolving since life's beginning, viruses have established the ability to efficiently infect and transfer their genomes in a wide variety of mammalian cell types. Likewise, and generally considered beneficial for survival, mammals have equally evolved highly complex mechanisms to protect themselves against invading pathogens such as viral gene transfer vectors. However, it is this protective immune response that often presents major obstacles for successful long-term therapy. Fortunately, the gene transfer community has been extensively studying the mechanisms of immune responses against viral vectors and has started to develop strategies and protocols to block or circumvent such responses. In this Research Topic, the editors present a collection of mini-Reviews, in-depth Reviews, Perspective, and Primary research articles that highlight both the well established and emerging strategies currently being used in blocking the immune response to gene transfer with viral vectors.

Viral vectors such as adenovirus and adenovirus-associated virus (AAV) are superior tools for gene therapy due to their high efficiency of transduction into a variety of cells in vivo. Administration of viral vectors often provokes the initiation of innate and antigen-specific adaptive immune responses against the virus and/or the therapeutic transgene products. Activation of these pathways elicits a flurry of antiviral and pro-inflammatory signals that can recruit effector lymphocytes, inhibit viral transduction, and encourage elimination of transduced cells over a period of time. Additionally, the de novo expression of a wild-type protein may trigger an adaptive immune response in the form of neutralizing antibodies. Likewise, pre-existing immunity (immunological memory) to the gene transfer vector resulting from prior exposure to the virus often prevents efficient gene transfer. All of these scenarios pose serious hurdles for successful gene therapy.

The first review article of this special topic provides a comprehensive overview of the innate immune responses to AAV. The authors highlight and discuss recent discoveries regarding strategies to abrogate potentially detrimental signaling pathways (Rogers et al., 2011). Historically, the innate immune response to singlestranded AAVs has been considered weak and transient when compared to the potent and prolonged response elicited by Ad vectors, thus prompting many investigators to focus more on the adaptive immune response to AAV. In more recent years our understanding of the early innate mechanisms of immune responses to viral vectors has greatly improved. The authors present an up-to-date analysis of the mechanisms surrounding the innate immune response to single-stranded and self-complimentary AAVs, including the role of the viral capsid, the effects on target tissue, and the therapeutic potential of blocking innate responses.

An equally impressive review detailing the current understanding relative to Ad vector mediated induction of the innate and adaptive immune responses is presented by Aldhamen et al. (2011). Ad vectors possess several advantages, the most important of which is that they can be easily, and routinely produced to high titers, however they also rapidly activate innate immune responses as well as potent cellular and humoral adaptive immune responses. This review examines the impact these responses have on the safety and efficacy of Ad vectorbased therapies (Aldhamen et al., 2011).
They also highlight strategies proposed to either mitigate or harness Ad-induced innate and adaptive immune responses for the improved and broadened development of advanced, Ad-based therapies.

Adenovirus-associated virus vectors have shown considerable promise as a gene delivery tool for clinical gene therapy applications. Unfortunately, the presence of pre-formed neutralizing antibodies directed against the AAV capsid in a large proportion of the human population due to widespread prior exposure to the wild-type virus has prevented AAV from reaching its full potential as a gene therapy vector. Bartel et al. (2011) provide an intriguing review that examines the use of capsid engineering as a means to evade pre-existing immunity. The authors discuss the consequences of humoral anti-AAV immune responses and potential strategies to prevent them. Additionally, Arnett et al. (2011) discusses the influence that cross reactivity of preexisting antibodies has on gene therapy, including neonatal administration of viral vectors as a means to circumvent such antibody responses.

As a target tissue for gene therapy, muscle is appealing because it is abundant, easily accessible, and procedures involving gene transfer to muscle are relatively safe and non-invasive (Hoffman et al., 2007). The mini-review by Wang et al. focuses on recent reports of immunity to AAV capsid proteins and transgene products in the context of gene delivery to muscles for treating both muscular dystrophies and other non-muscle diseases. They provide strategies of immune modulation and tolerance induction in order to prevent unwanted immune responses to the vector and/or the therapeutic gene product (Wang et al., 2011). Additionally, the research article presented by the Boyer group, evaluates a treatment protocol designed to inhibit the deleterious immune activation during muscle gene transfer. Their strategy 
is based on the administration of CTLA-4/ Ig in order to block the co-stimulatory signals required early during immune priming combined with gene transfer of PD-1 ligands to inhibit $\mathrm{T}$ cell functions at the tissue sites (Adriouch et al., 2011).

It has become evident that the manipulation of various co-stimulatory pathways to regulate host immune responses is of therapeutic interest. Addressing this, Huang and Yang, offer a broad review of relevant $\mathrm{T}$ cell co-stimulatory pathways in the activation of both $\mathrm{T}$ and B-cells, and provide strategies for targeting these co-stimulatory pathways in gene therapy applications. Ultimately, they suggest that studies should focus on targeting multiple pathways including both the positive and negative co-stimulatory pathways (Huang and Yang, 2011). Using a multiple pathway approach in an animal model of hemophilia, the Herzog lab presents new findings that suggest that transient immune modulation using a cocktail of rapamycin, IL-10, and specific peptides could prevent or possibly reverse gene therapy-induced inhibitor formation (Nayak et al., 2011).

The success of in vivo gene therapy not only depends on the ability to control the immune response toward the input vector, but also to the therapeutic transgene. Using both vector-based and pharmacological approaches, various groups have explored various approaches to control the immunemediated clearance of transgene-expressing cells after viral delivery. One approach using micro-RNA transgene regulation to generate a tolerogenic response is reviewed by Goudy et al. They further speculate on possible mechanisms used by the liver to induce the transgene-specific regulatory $\mathrm{T}$ cells (Goudy et al., 2011). Another method to modulate tolerance induction is via gene transfer to B-cells and such an approach is presented by $\mathrm{Su}$ et al. Here, the authors demonstrate that host IL-10 is critical for the tolerogenicity of B-cell based peptideIgG gene therapy (Su et al., 2011).

Overall, this Research Topic: "Approaches to Blocking the Immune Response to Gene Transfer with Viral Vectors" provides a welldeveloped overview of the current therapeutic potentials of viral gene therapy. This collection of articles not only provides a dynamic review and analyses of the complex immune responses present in current gene therapy applications, but they also provide insight for the future direction of the field.

\section{REFERENCES}

Adriouch, S., Franck, E., Drouot, L., Bonneau, C., Jolinon, N., Salvetti, A., and Boyer, O. (2011). Improved immunological tolerance following combination therapy with CTLA-4/Ig and AAV-mediated PD-L1/2 muscle gene transfer. Front. Microbiol. 2:199. doi: 10.3389/ fmicb.2011.00199

Aldhamen, Y.A., Seregin, S. S., and Amalfitano, A. (2011) Immune recognition of gene transfer vectors: focus on adenovirus as a paradigm. Front. Microbiol. 2:40. doi: 10.3389/fimmu.2011.00040

Arnett, A. L. H., Garikipati, D., Wang, Z., Tapscott, S., and Chamberlain, J. S. (2011). Immune responses to rAAV6: the influence of canine parvovirus vaccination and neonatal administration of viral vector. Front. Microbiol. 2:220. doi: 10.3389/fmicb.2011.00220

Bartel, M., Schaffer, D., and Büning, H. (2011). Enhancing the clinical potential of AAV vectors by capsid engineering to evade pre-existing immunity. Front. Microbiol. 2:204. doi: 10.3389/fmicb.2011.00204

Goff, S. P., and Berg, P. (1976). Construction of hybrid viruses containing SV40 and $\lambda$ phage DNA segments and their propagation in cultured monkey cells. Cell 9, 695-705.
Goudy, K. S., Annoni, A., Naldini, L., and Roncarolo, M.-G. (2011). Manipulating immune tolerance with micro-RNA regulated gene therapy. Front. Microbiol. 2:221. doi: 10.3389/fmicb.2011.00221

Hoffman, B. E., Dobrzynski, E., Wang, L., Hirao, L., Mingozzi, F., Cao, O., and Herzog, R. W. (2007). Muscle as a target for supplementary factor IX gene transfer. Hum. Gene Ther. 18, 603-613.

Huang, X., and Yang, Y. (2011). Targeting co-stimulatory pathways in gene therapy. Front. Microbiol. 2:202. doi: 10.3389/fmicb.2011.00202

Li, H., and Ertl, H. C. J. (2011). Can viruses be modified to achieve sustained gene transfer. Front. Microbiol. 2:152. doi: 10.3389/fmicb.2011.00152

Nayak, S., Sarkar, D., Perrin, G. Q., Moghimi, B., Hoffman, B. E., Zhou, S., Byrne, B. J., and Herzog, R. W. (2011). Prevention and reversal of antibody responses against factor IX in gene therapy for hemophilia B. Front. Microbiol. 2:244. doi: 10.3389/fmicb.2011.00244

Rogers, G. L., Martino, A. T., Aslanidi, G. V., Jayandharan, G. R., Srivastava, A., and Herzog, R. W. (2011). Innate immune responses to AAV vectors. Front. Microbiol. 2:194. doi: 10.3389/fmicb.2011.00194

Su,Y.,Zhang,A.H.,Noben-Trauth,N., and Scott,D.W.(2011). B-cell gene therapy for tolerance induction: host but not donor B-cell derived IL-10 is necessary for tolerance. Front. Microbiol. 2:154. doi: 10.3389/fmicb.2011.00154

Wang, Z., Tapscott, S. J., Chamberlain, J. S., and Storb, R. (2011). Immunity and AAV-mediated gene therapy for muscular dystrophies in large animal models and human trials. Front. Microbiol. 2:201. doi: 10.3389/ fmicb.2011.00201

Received: 18 April 2012; accepted: 01 May 2012; published online: 24 May 2012.

Citation: Hoffman BE, Ertl HCJ, Terhorst C, High KA and Herzog RW (2012) Gene therapy research at the Frontiers of viral immunology. Front. Microbio. 3:182. doi: 10.3389/ fmicb.2012.00182

This article was submitted to Frontiers in Microbial Immunology, a specialty of Frontiers in Microbiology. Copyright (C) 2012 Hoffman, Ertl, Terhorst, High and Herzog. This is an open-access article distributed under the terms of the Creative Commons Attribution Non Commercial License, which permits non-commercial use, distribution, and reproduction in other forums, provided the original authors and source are credited. 